\title{
Efeitos da estimulação diafragmática transcutânea sincronizada em pacientes com doença pulmonar obstrutiva crônica (DPOC): um estudo piloto
}

\author{
Percy Nohama*, Rafaela Fernanda Jorge, Marcelo Henrique Valenga
}

\begin{abstract}
Resumo A pesquisa descrita neste artigo teve por objetivo avaliar quali-quantitativamente os efeitos da estimulação diafragmática transcutânea sincronizada em portadores de DPOC. Para isso, desenvolveu-se um sistema de estimulação elétrica controlado pelo sinal respiratório, a partir das variações de temperatura durante os eventos de inspiração e expiração, medidas por meio de dois termistores do tipo NTC inseridos dos lados interno e externo de uma máscara de respirador. Seis voluntários portadores de DPOC, de ambos os sexos, com idade entre 56 e 71 anos, foram submetidos a 10 sessões estimulatórias de 20 minutos. O padrão do sinal estimulatório continha pulsos com duração de $90 \mu$ s e repouso de $400 \mu \mathrm{s}$, gerados em intervalos regulares e modulados em bursts com período ativo de $1470 \mu \mathrm{s}$ e inativo de $600 \mu \mathrm{s}$, apresentando perfil trapezoidal com tempos de subida, descida e platô de $500 \mathrm{~ms}$ cada. Todos os pacientes foram submetidos a uma avaliação inicial contendo: teste de força muscular respiratória avaliada por meio de $\mathrm{PI}_{\text {máx }}$ e $\mathrm{PE}_{\text {máx }}$, teste de função pulmonar e aplicação do questionário de qualidade de vida SGRQ. Após 10 sessões, houve um aumento na força muscular inspiratória em todos os pacientes, onde a $\mathrm{PI}_{\text {máx }}$ sofreu um incremento médio de 66,67 $\pm 12,11 \mathrm{cmH}_{2} \mathrm{O}$ para $91,67 \pm 25,03 \mathrm{cmH}_{2} \mathrm{O}$, a $\mathrm{PE}_{\text {máx }}$ de $92,50 \pm 10,84 \mathrm{cmH}_{2} \mathrm{O}$ para $116,67 \pm 8,16 \mathrm{cmH}_{2} \mathrm{O}$. Também observou-se melhora da qualidade de vida no domínio sintoma, de 49,10 $\pm 19,40$ para 28,60 $\pm 25,20$; no domínio atividade, de $83,40 \pm 12,50$ para $67,57 \pm 18,80$; no domínio impacto, de $54,10 \pm 11,34$ para $38,00 \pm 27,07$; e escore total, de $65,50 \pm 7,60$ para 44,47 $\pm 22,31$. A partir desses resultados, concluiu-se que a estimulação elétrica diafragmática sincronizada pode promover resultados positivos em portadores de DPOC.
\end{abstract}

Palavras-chave Estimulação elétrica diafragmática sincronizada, Estimulação elétrica neuromuscular, Eletroventilação, Disfunção diafragmática, DPOC.

\section{Effects of transcutaneous diaphragmatic synchronized pacing in patients with chronic obstructive pulmonary disease (COPD)}

Abstract The research described in this paper has the goal of evaluating the effects of transcutaneous diaphragmatic synchronized pacing in patients with COPD. In order to achieve the experimental protocol, it has been developed a system for electrical stimulation triggered by the respiratory signal obtained from the temperature variations during inspiration and expiration events, using two NTC thermistors coupled inside and outside of a respirator mask. It was studied six volunteers with COPD, of both sexes, aged between 56 to 71 years, who were submitted to 10 sessions of 20 minutes. The stimulatory parameters were set to: active phase of the pulse of $90 \mu \mathrm{s}$, and inactive phase of $400 \mu \mathrm{s}$, generated at regular intervals and modulated in bursts with active and inactive periods of $1470 \mu$ s and $600 \mu$ s, respectively; presenting trapezoidal envelope with rise time, fall time and plateau of $500 \mathrm{~ms}$. All patients underwent an initial assessment including: test of respiratory muscle strength seen through the MIP and MEP tests, lung function test and application of $S G R Q$ quality of life questionnaire. After 10 sessions, there is an increasing inspiratory muscle strength in all patients, where MIP has an average increase of $66.67 \pm 12.11 \mathrm{cmH}_{2} \mathrm{O}$ to $91.67 \pm 25.03 \mathrm{cmH}_{2} \mathrm{O}, \mathrm{MEP}$ from $92.50 \pm 10.84 \mathrm{cmH}_{2} \mathrm{O}$ to $116.67 \pm 8.16 \mathrm{cmH}_{2} \mathrm{O}$, and also observed a better quality of life in the symptom domain of $49.10 \pm 19.40$ to $28.60 \pm 25.20$, in the activity domain of $83.40 \pm 12.50$ to $67.57 \pm 18.80$, and in the impact domain of $54.10 \pm 11.34$ to $38.00 \pm 27.07$ and total score of $65.50 \pm 7.60$ to $44.47 \pm 22.31$. Therefore, we concluded that the transcutaneous diaphragmatic synchronized pacing can promote positive outcomes in patients with COPD.

Keywords Transcutaneous diaphragmatic synchronized pacing, Neuromuscular electrical stimulation, Electroventilation, Diaphragmatic dysfunction, COPD. 


\section{Extended Abstract}

\section{Introduction}

Various types of diaphragmatic dysfunction may lead to a change of its operation, among them, the COPD, which is characterized by the chronic airflow limitation, triggering changes in ventilatory mechanics. Currently, many electroventilatory methods have been used in an attempt to recover the functionality of diaphragm muscle, among them the diaphragmatic electrical stimulation. The aim of this research is the evaluation of the effects of transcutaneous synchronized diaphragmatic pacing in patients with moderate to severe COPD, through qualitative and quantitative assessment of the recruitment of diaphragm muscle and the impact of treatment on quality of their life.

\section{Material and Methods}

In order to achieve the experimental protocol, it had been developed a system for electrical stimulation triggered by the respiratory signal. This signal comes from the changes in temperature events of inspiration and expiration, through two NTC thermistors coupled inside and outside of a respirator mask. Six volunteers with moderate and severe COPD participated of this experimental research, of both genders, aged between 56 to 71 years, which were submitted to 10 sessions of 20 minutes each. The stimulatory parameters were set to: active phase of the pulse of $90 \mu \mathrm{s}$, and inactive phase of $400 \mu \mathrm{s}$, generated at regular intervals $(2.04 \mathrm{kHz})$ and modulated in bursts with active and inactive periods of $1470 \mu \mathrm{s}$ and $600 \mu \mathrm{s}(483 \mathrm{~Hz})$, respectively; presenting trapezoidal envelope with rise time, fall time and plateau of $500 \mathrm{~ms}$. All patients underwent an initial assessment including: test of respiratory muscle strength measured trough maximum inspiratory pressure (MIP) and maximum expiratory pressure (MEP), lung function test and application of SGRQ quality of life questionnaire.

\section{Results}

After 10 sessions, it was observed an increment on the inspiratory muscle strength in all patients. MIP had an average increase of $25.00 \pm 13.78 \mathrm{cmH}_{2} \mathrm{O}$ (from $66.67 \pm 12.11$ to $\left.91.67 \pm 25.03 \mathrm{cmH}_{2} \mathrm{O}\right), \mathrm{MEP}$ showed an average increase of $24.17 \pm 9.17 \mathrm{cmH}_{2} \mathrm{O}$ (from $92.50 \pm 10.84$ to $116.67 \pm 8.16 \mathrm{cmH}_{2} \mathrm{O}$ ), and also observed a better quality of life in the symptom domain of $49.10 \pm 19.40$ to $28.60 \pm 25.20$, in the activity domain of $83.40 \pm 12.50$ to $67.57 \pm 18.80$, and in the impact domain of $54.10 \pm 11.34$ to $38.00 \pm 27.07$ and total score of $65.50 \pm 7.60$ to $44.47 \pm 22.31$.

\section{Conclusion}

The synchronized electrical stimulation promotes the increasing of diaphragmatic muscle strength in patients with COPD, reflecting in positive results as regards the respiratory muscle strength and the improvement of life quality. 


\section{Introdução}

A doença pulmonar obstrutiva crônica (DPOC) vem sendo considerada um problema de conotação médica e social de primeira magnitude, provocando altos índices de mortalidade e morbidade mundial nos últimos tempos (Kunikoshita et al., 2006; Nishimura et al., 2002; Sociedade..., 2000).

O termo DPOC compreende um grupo de características clínico-patológicas que têm em comum a obstrução ao fluxo aéreo expiratório, denominadas enfisema pulmonar e bronquite crônica. Diante dessa obstrução pulmonar, os portadores de DPOC sofrem prejuízos na mecânica pulmonar e na musculatura periférica, de modo progressivo, persistente e irreversível (American..., 1995; Global..., 2001, 2006).

A predominância dessas alterações varia de indivíduo para indivíduo e está diretamente relacionada a sinais e sintomas apresentados, tais como: tosse, produção de secreção, limitação do fluxo aéreo, dispnéia e dificuldades nas trocas gasosas (American..., 1995; Grosselink et al., 1997; Sarmiento-Ramírez et al., 2002).

O curso progressivo e crônico da doença é agravado por períodos de aumento desses sintomas. Quando o tratamento ambulatorial não é eficaz, pode haver necessidade de internação hospitalar, que é o maior determinante de custo da DPOC (Miravitles, 2004).

A obstrução pulmonar do fluxo expiratório é causada por diversos fatores fisiopatológicos que levam à hiperinsulflação pulmonar. Tais processos tendem a gerar uma diminuição da capacidade inspiratória, devido à dispnéia que inicialmente surge durante a atividade física e, com a progressão da doença, também no repouso (Celli e MacNee, 2004).

Esse fato, além de promover alterações de volumes e da capacidade pulmonar, interfere na atuação, mobilidade e conformidade do diafragma, que perde seu formato de cúpula e tende a se retificar, diminuindo sua zona de aposição e limitando a ação ventilatória, normalmente mais evidente na porção inferior da caixa torácica. Isso pode levar o paciente a uma respiração paradoxal decorrente da descoordenação entre os compartimentos torácico e abdominal (Yamaguti et al., 2009).

Na prática clínica, observa-se que em estados mais avançados, o portador de DPOC possui restrições para realizar alguns tipos de exercícios, fazendo com que interrompam o tratamento e, consequentemente, mantendo os problemas impostos por esta doença (Celli e MacNee, 2004).

Por outro lado, inúmeros métodos eletroventilatórios vêm sendo usados para reabilitar o músculo diafragmático e melhorar a ventilação pulmonar em pacientes que apresentam distúrbios respiratórios. Esta técnica consiste em aplicar trens de pulsos nos pontos motores do nervo frênico (responsável pela inervação do músculo diafragmático) com o uso de eletrodos de superfície posicionados na região do sexto, sétimo e oitavo espaços intercostais, região paraxifóidea, ou na base do pescoço, entre o músculo escaleno e o esternocleidomastóide (Nishimura et al., 2002; Sociedade..., 2000).

Sabe-se que a eletroestimulação pode promover melhorias na ventilação pulmonar associada ao fortalecimento muscular diafragmático (Valenga et al., 2008). Por isso, esse método oferece perspectivas promissoras em patologias que por um motivo fisiológico específico comprometeram o trabalho e a força do músculo diafragma, como em situações em que ocorre invasão tumoral por neoplasias pulmonares, compressão ou invasão por neoplasias mediastínicas, lesão ou ressecção cirúrgica, comprometimento das raízes por artrose cervical, doenças neurológicas como a poliomiosite, DPOC, neuropatias, miopatias, disfunções diafragmáticas pós-cirúrgicas em revascularização do miocárdio ou em tetraplegia, além de diminuição do tempo requerido para o desmame em casos da ventilação mecânica prolongada, principal motivo de atrofia muscular por desuso (Cancelierro et al., 2006; Hausson e Celli, 2000; Forti et al., 2005).

Grandes são as variações protocolares dos programas de reabilitação pulmonar, o que dificulta o estabelecimento padrão para a aplicação em portadores de DPOC (Costa, 1999). Na prática clínica, em estágios mais avançados da doença, os pacientes apresentam disfunção muscular periférica, o que os restringem na realização de alguns tipos de treinamento, fazendo com que o desestímulo os leve, não poucas vezes, a interromper o tratamento (Dourado e Godoy, 2004). Tal síndrome periférica não acomete o músculo diafragma, porém eles possuem limitação da capacidade ventilatória imposta pela obstrução ao fluxo expiratório.

Poucos são os estudos relacionados à estimulação elétrica diafragmática sincronizada (EEDS) com o movimento respiratório natural. Ademais, essa técnica constitui-se numa opção inovadora de reabilitação diafragmática e na capacidade ventilatória, promovendo um melhor desempenho no recrutamento muscular, potencializando a fase inspiratória através da estimulação elétrica, além de facilitar o procedimento, propiciando o acompanhamento do paciente pelo staff clínico durante o tratamento, o que não ocorre quando aplicado um protocolo de estimulação diafragmática convencional (Biaggi et al., 2000).

Ávila (2001), por meio de um estudo empregando a técnica de estimulação elétrica diafragmática sincronizada com o sinal respiratório via sensor à 
fibra óptica, em seis indivíduos saudáveis, obteve resultados estatisticamente satisfatórios no que diz respeito à força muscular inspiratória e expiratória.

Assim, os objetivos do estudo descrito neste artigo consistem em apresentar a instrumentação desenvolvida e discutir os resultados desta pesquisa experimental, na qual se avaliam os efeitos da estimulação diafragmática transcutânea sincronizada em portadores de DPOC com referência à força muscular inspiratória e expiratória, e seu impacto na qualidade de vida desses pacientes.

\section{Metodologia}

\section{Sensor empregado no sistema de sincronismo do sinal respiratório}

Para detectar o sinal produzido durante a respiração, utilizou-se o sensor de temperatura M20-B-101, um termistor do tipo NTC produzido pela empresa brasileira ADD-THERM, que pode ser observado na Figura 1, com resistência de $30 \Omega$ à temperatura de $25^{\circ} \mathrm{C}$ e cuja curva característica pode ser vista na Figura 2.

\section{Sistema de aquisição do sinal respiratório}

Para aquisição do sinal respiratório, optou-se pela utilização de dois termistores com o intuito de compensar as variações de temperatura. Um dos sensores foi acoplado na parte interna de uma máscara respiratória, em contato direto com o fluxo de ar inspirado e expirado pelas cavidades bucal e nasal, e o segundo sensor foi posicionado na parte externa da máscara e sujeito à temperatura ambiente, como ilustrado na Figura 3a.

Por meio de um amplificador diferencial, foi possível amplificar a diferença de potencial proporcional à diferença de temperatura entre os termistores, como ilustrado na Figura 3b, gerando um sinal de tensão que expressa a magnitude e a direção do fluxo nas vias respiratórias. Esse sinal é amplificado

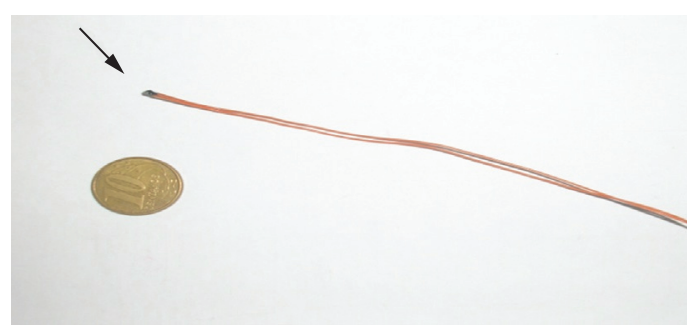

Figura 1. Foto do sensor NTC M20-B-101 e comparativo de sua dimensão com uma moeda de $\mathrm{R} \$ 0,10$.

Figure 1. Photo of the NTC M20-B-101 sensor and comparison of its size with a $R \$ 0.10$ coin aproximadamente 100 vezes, fornecendo uma faixa de saída entre 0 e $5 \mathrm{~V}$, visto que sua amplitude original é inadequada para a conversão analógico-digital.

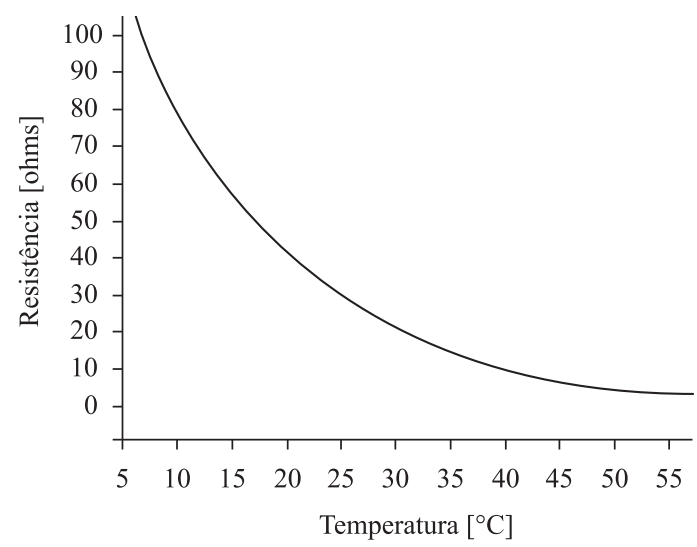

Figura 2. Curva característica do termistor NTC M20-B-101 que em $25^{\circ} \mathrm{C}$ apresenta resistência de $30 \Omega$.

Figure 2. NTC M20-B-101 thermistor characteristic curve. At $25^{\circ} \mathrm{C}$ its resistance is $30 \Omega$.
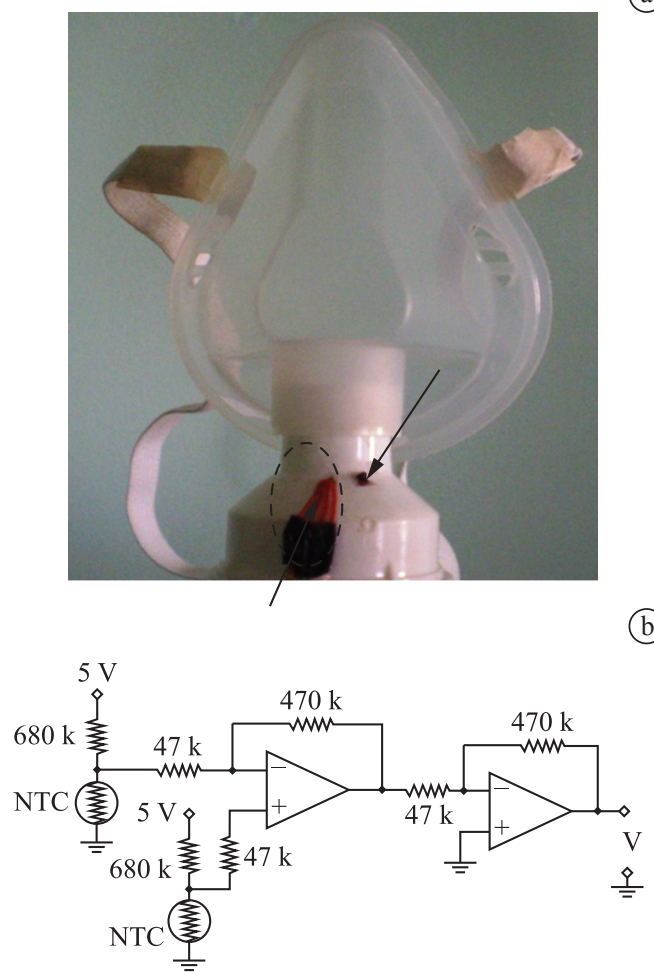

Figura 3. a) máscara respiratória contendo os sensores posicionados interna e externamente à máscara. b) amplificador diferencial utilizado para determinar a diferença de temperatura entre os dois termistores posicionados dentro e fora da máscara respiratória.

Figure 3. a) breathing mask containing the sensors placed inside and outside the mask. b) differential amplifier used to measure the temperature difference between the two thermistors positioned inside and outside the respirator mask. 
Em seguida, e conforme ilustrado na Figura 4, efetua-se a digitalização deste sinal através de um conversor analógico-digital controlado por um microcontrolador PIC16F876, programado para fazer a aquisição a uma taxa de 500 amostras por segundo. O conversor A/D utilizado é um periférico do microcontrolador com resolução de 10 bits. No entanto, só foram considerados os 8 bits mais significativos da conversão realizada, eliminando os dois bits que contêm as informações das menores variações de amplitude no sinal analógico convertido, que no caso concreto é insignificante. Essa supressão de dois bits colaborou para a geração de um sinal gráfico menos "ruidoso". Depois de cada conversão, o sinal digital é enviado para o computador através da porta serial. Foi utilizada uma frequência de aquisição elevada porque um dos objetivos dessa conversão era proporcionar ao corpo clínico um retorno visual da variação da temperatura nas vias aéreas (a qual reflete as variações respiratórias), através do software desenvolvido em plataforma Labview ${ }^{\circledR}$ (National Instruments). Assim, seria possível observar variações respiratórias rápidas, como no caso de um evento de tosse, com uma boa resolução. Poder-se-ia ter utilizado uma frequência de aquisição menor se o objetivo fosse apenas calcular os instantes de disparo do estimulador muscular.

Depois de sucessivas leituras, as amostras do sinal respiratório são digitalizadas e comparadas com os valores anteriores a um ponto máximo ou mínimo, segundo as referências estabelecidas na aquisição do movimento respiratório anterior. A verificação

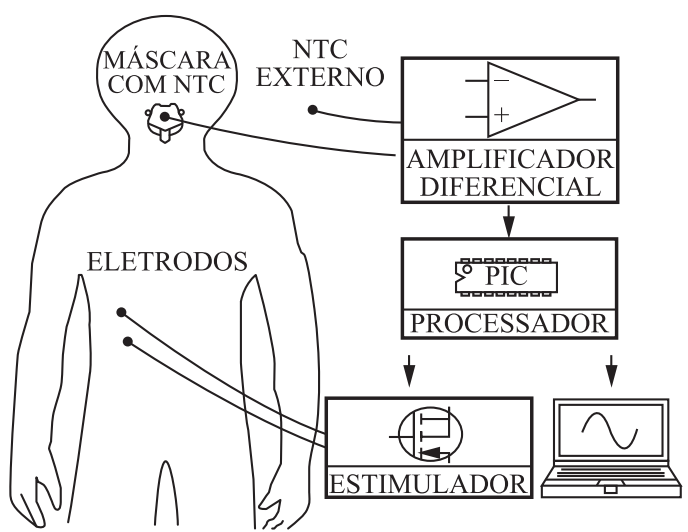

Figura 4. Diagrama funcional do sistema desenvolvido. O processador utiliza o sinal amplificado dos termistores para determinar os momentos exatos de disparo do estimulador. Para isso, os eletrodos são posicionados nos pontos motores da musculatura envolvida no processo respiratório.

Figure 4. Functional diagram of the developed system. The processor uses the amplified signal of the thermistors to determine the exact moments of firing of the stimulator. For this application, the electrodes are positioned on the motor points of muscles involved in breathing process. dos instantes de máximo e de mínimo para a decisão do momento de disparo de um estimulador elétrico muscular é efetuada pelo microcontrolador, seguindo dois critérios:

- Ponto de mínimo (final da fase inspiratória e início da fase expiratória): 1) quando a derivada do sinal deixa de ser negativa (decrescente) e passa a ser positiva (crescente), indicando um sinal decrescente; 2) quando o valor da amostra atual for menor que o limiar fixado em $15 \%$ da amplitude do ciclo respiratório anterior;

- Ponto de máximo (final da fase expiratória e início da fase inspiratória): 1) quando a derivada do sinal deixar de ser positiva (crescente) e passar a ser negativa (decrescente), indicando um sinal crescente; 2) quando o valor da amostra atual for maior que o limiar fixado em $85 \%$ da amplitude do ciclo respiratório anterior.

Quando uma das duas condições é satisfeita, o microcontrolador altera o nível lógico de saída de uma das suas portas digitais (PORTC-3), que ligada a um driver aciona um estimulador elétrico neuromuscular desenvolvido no Laboratório de Engenharia de Reabilitação da UTFPR (Biaggi et al., 2000), por meio da comutação de um micro-relé. A utilização de chaveamento eletromecânico possibilita a isolação elétrica entre o sistema de detecção e o circuito de estimulação, sendo este alimentado por bateria, garantindo a isolação do paciente (Webster, 1998).

$\mathrm{O}$ microcontrolador foi programado para enviar à sua porta serial (interface RS-232) o valor digitalizado de cada amostra do sinal respiratório. A disponibilidade desse sinal permitiu o desenvolvimento de um aplicativo em plataforma Labview, que interpreta as amostras do sinal respiratório na porta serial e o mostra na tela do monitor de um computador, na forma gráfica, como ilustrado na Figura 5a. Esse aplicativo possibilita que um profissional da área médica, utilizando um notebook, monitore e controle todo o processo e, ainda, avalie seu desempenho ao longo do tempo.

Após a realização de testes in vivo, constatou-se que o sistema de sincronismo apresentou sensibilidade adequada às pequenas variações de deslocamento de ar e uma rápida resposta no tempo, viabilizando sua utilização com diferentes padrões respiratórios, como o exemplo ilustrado na Figura 5a. Nesta figura, podem-se observar também os marcadores de disparo do estimulador, nos instantes definidos como início dos eventos de inspiração e expiração. Na pesquisa experimental aqui descrita empregou-se apenas o pulso de disparo do evento inspiração. 


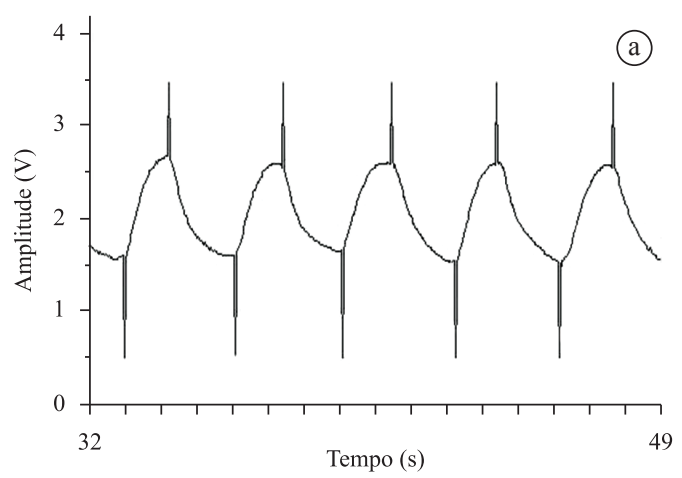

(b)

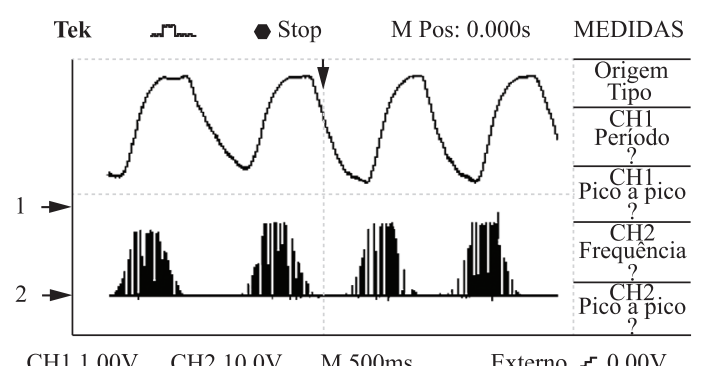

Figura 5. a) visualização do sinal respiratório, amplitude $(\mathrm{V}) \times$ tempo $(\mathrm{s})$, adquirido através da interface serial implementada na plataforma Labview. Os picos de tensão nos pontos máximos e mínimos indicam os instantes em que o estimulador pode ser ativado. b) tela de um osciloscópio mostrando o sincronismo entre o final da fase expiratória (amplitude mínima) e o disparo dos pulsos do estimulador elétrico diafragmático. $\mathrm{O}$ eixo das ordenadas representa a diferença de potencial $(\mathrm{V})$ que representa o sinal repiratório e o eixo das abscissas, o tempo (ms).

Figure 5. a) visualization of the respiratory signal, amplitude $(V) \times$ time $(s)$ acquired through the computer serial interface and implemented in Labview Platform. The peaks over the maximum and minimum points indicate the moments when the stimulator can be activated. b) an oscilloscope screen showing the timing between the end of the expiratory phase (minimum amplitude) and the firing pulses of electrical diafragmatic stimulator. The ordinate axis represents the potential difference $(V)$ which corresponds to the respiratory signal and the abscissa axis represents time (ms).

O programa desenvolvido na plataforma Labview tem ainda a função de enviar, através da porta serial, o comando para o microcontrolador ativar o estimulador elétrico muscular. Na Figura 5b, ilustra-se a forma de onda estimulatória e o sinal respiratório que controla o início do estímulo, obtida com auxílio de um osciloscópio Tektronix, modelo TDS 1000.

\section{Participantes}

Seis portadores de DPOC foram selecionados, os quais apresentavam obstrução moderada e grave, sintomas de dispnéia e diminuição da pressão máxima inspiratória e expiratória, com diagnóstico estabelecido há pelo menos seis meses, de ambos os sexos, com idade entre 56 a 71 anos, nos quais a relação $\mathrm{VEF}_{1} /$ $\mathrm{CVF}$ e o $\mathrm{VEF}_{1}$ eram inferiores a $60 \%$. Os pacientes foram agendados pela equipe médica de pneumologia do Hospital Universitário Cajuru, Curitiba, Brasil. As avaliações e os atendimentos foram efetuados no setor de Métodos Gráficos do próprio hospital.

O estudo foi realizado com a aprovação do Comitê de Ética em Pesquisa envolvendo Seres Humanos da Pontifícia Universidade Católica do Paraná, sob registro $n^{\circ}$ 0001497/08.

Os voluntários formalizaram a participação na pesquisa por meio de um termo de consentimento informado por escrito. Incluíram-se no estudo os portadores de DPOC, entre 56 a 71 anos, estando sob tratamento medicamentoso otimizado, apresentando diminuição dos níveis de pressão inspiratória máxima ( $\left.\mathrm{PI}_{\max }\right)$ e pressão expiratória máxima $\left(\mathrm{PE}_{\max }\right)$, comprometimento da qualidade de vida. Foram excluídos todos os pacientes portadores de outras pneumopatias e/ou cardiopatias graves, alterações cognitivas importantes, os que haviam participado de um programa de reabilitação nos últimos dois meses e os que não puderam comparecer regularmente ao atendimento fisioterápico.

\section{Protocolo de avaliação}

Os seis pacientes selecionados foram submetidos a uma avaliação inicial composta por anamnese, antecedentes pessoais, exame físico, aferição dos sinais vitais (PA, FC, FR, $\mathrm{SatO}_{2}$ ) e da Escala de Borg Modificada, que é específica para dispnéia, além dos testes de:

- Função pulmonar: utilizando um espirômetro, com o qual foram realizados três testes para definir o grau de obstrução pulmonar, e onde se considerou somente o maior valor obtido entre eles. O resultado determinado pelo teste foi comparado com valores de referência previamente descritos na literatura (American..., 1995; Knudson et al., 1983; Sociedade..., 2000);

- Força muscular inspiratória e expiratória: utilizando o mano vacuômetro M-120, fabricado pela Comercial Médica, com faixa operacional de $120 \mathrm{cmH}_{2} \mathrm{O}$. As pressões inspiratória $\left(\mathrm{PI}_{\text {máx }}\right)$ e expiratória $\left(\mathrm{PE}_{\text {máx }}\right)$ máximas servem como indicadores da força muscular respiratória (Back e Hyatt, 1969) e, por isso, foram utilizadas. As medidas foram efetuadas com o paciente na posição sentada, com uma pinça nasal colocada nas narinas do voluntário, a fim de evitar que inspirasse pela cavidade nasal no momento do teste. Cada paciente era orientado a respirar através de um bocal firmemente posicionado para evitar vazamento perioral. A 
$\mathrm{PI}_{\text {max }}$ foi obtida a partir da inspiração máxima e a $\mathrm{PE}_{\text {máx }}$ a partir da expiração máxima. Foram realizadas três manobras para as medidas e selecionado o melhor valor obtido (Fiz et al., 1989). Os valores obtidos por cada voluntário foram comparados aos seus respectivos valores previstos para a população brasileira, de acordo com as seguintes equações de predição propostas por Neder et al. (1999):

\section{Homens}

$$
\begin{aligned}
& P I_{\text {máx }}=-0,8 \times(\text { idade })+155,3 \\
& P E_{\text {máx }}=-0,81 \times(\text { idade })+165,3
\end{aligned}
$$

Mulheres

$$
\begin{aligned}
& P I_{\text {máx }}=-0,49 \times(\text { idade })+110,4 \\
& P E_{\text {máx }}=-0,61 \times(\text { idade })=115,6
\end{aligned}
$$

- Qualidade de vida: avaliada por meio do questionário de qualidade de vida e dispnéia, neste caso, o St. George Respiratory Questionnaire (SGRQ), específico aos portadores de doenças respiratórias, o qual foi aplicado por um entrevistador devidamente treinado, com o intuito de facilitar a compreensão do enunciado, sem interferir na resposta do paciente. O SGRQ é composto por 76 questões divididas em três domínios: sintoma, atividade e impacto, sendo que cada item apresenta um valor pré-determinado (Camelier et al., 2006; Díez et al., 2005; Dourado et al., 2004; Jardim Junior et al., 2000; Rodrigues, 2003; Sousa et al., 2000). A partir daí, calculou-se o escore total que pode variar de 0 a 100 , que quanto menor o valor obtido, melhor a qualidade de vida do paciente.

\section{Treinamento}

Todos os pacientes foram submetidos a um protocolo composto por estimulação diafragmática sob a forma transcutânea realizada na posição Fowler (Azeredo, 1999). Para a aplicação da estimulação elétrica, aplicou-se um padrão estimulatório em que os pulsos apresentavam duração de $90 \mu$ s e repouso de $400 \mu \mathrm{s}$, gerados em intervalos regulares $(2,04 \mathrm{kHz})$, modulados por bursts com período ativo de $1470 \mu \mathrm{s}$ e inativo de $600 \mu \mathrm{s}(483 \mathrm{~Hz})$, conforme ilustrado na Figura 6a. A intensidade era ajustada de acordo com a sensibilidade de cada paciente que variava a cada aplicação. Além disso, a onda estimulatória possuía um perfil trapezoidal, com períodos de subida, platô e descida de $500 \mathrm{~ms}$, tal como indicado na Figura $6 \mathrm{~b}$.

Os estímulos elétricos foram aplicados por meio de eletrodos de superfície posicionados na região paraxifóidea, no ponto motor do nervo frênico, entre o sétimo e oitavo espaço intercostal (Geddes et al., 1988). Para se certificar que os eletrodos estavam posicionados adequadamente, eram disparados estímulos elétricos e, por meio de palpação (abaixo do processo xifóide), averiguava-se a contração muscular do diafragma (Ávila, 2001).

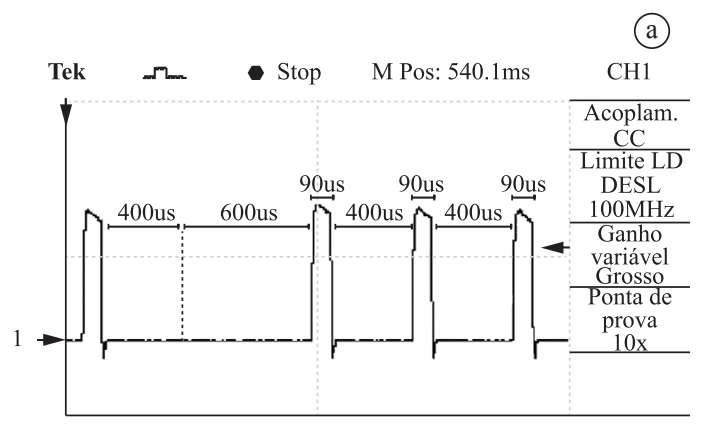

CH1 10.0V CH2 10.0VBw M 250us

$\mathrm{CH} 1$ ? $23.2 \mathrm{~V}$

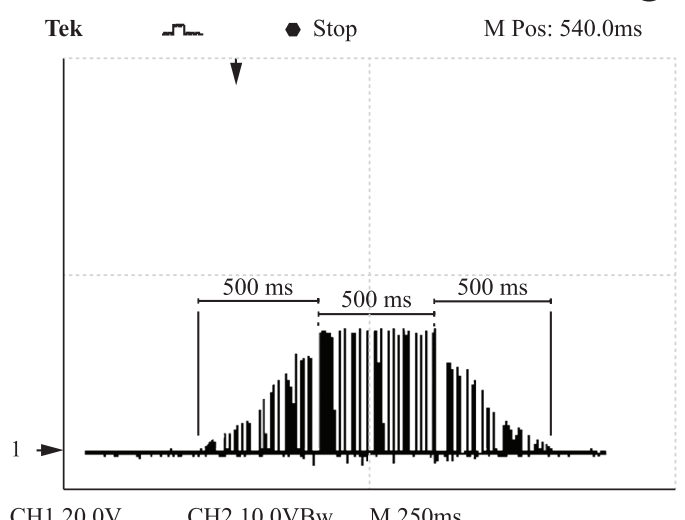

Figura 6. Ilustração da forma de onda do padrão estimulatório. (a) Os pulsos têm duração de $90 \mu$ s e repouso de $400 \mu$ s (frequência de $2,04 \mathrm{kHz}$ ) e são modulados em bursts com período ativo de $1470 \mu$ s e inativo de $600 \mu$ s (frequência de $483 \mathrm{~Hz}$ ), apresentando perfil trapezoidal com tempo de subida, tempo de descida e plateau de $500 \mathrm{~ms}$ cada (b). O eixo das ordenadas representa a amplitude do estímulo elétrico, em volts (V). A escala está graduada em $10 \mathrm{~V}(\mathrm{em}$ a) e $20 \mathrm{~V}$ (em b). $\mathrm{O}$ eixo das abscissas representa o tempo, ajustado em $250 \mu$ s (a) e em $250 \mathrm{~ms}$ (b), respectivamente.

Figure 6. Illustration of the stimulatory waveform pattern. (a) The pulses have a duration of $90 \mu$ s and $400 \mu$ s rest (frequency of $2.04 \mathrm{kHz}$ ) and are modulated in bursts with active period of $1470 \mu \mathrm{s}$ and $600 \mu \mathrm{s}$ of inactive one (frequency of $483 \mathrm{~Hz}$ ), with trapezoidal profile with rise time, fall time and plateau of $500 \mathrm{~ms}$ each (b). The ordinate axis represents the amplitude of the electrical stimuli in volts $(V)$. The scale is graduated at $10 \mathrm{~V}$ (a) and $20 \mathrm{~V}(\mathrm{~b})$, respectively. The abscissa axis represents time, set to $250 \mu \mathrm{s}$ (a) and $250 \mathrm{~ms}$ (b), respectively. 
Depois da confirmação do posicionamento correto do eletrodo sobre o ponto motor, solicitava-se ao paciente acoplar a máscara e, posteriormente, manter um padrão respiratório o mais regular possível para a decisão do momento de disparo.

Todos os pacientes participaram de um protocolo de estimulação elétrica diafragmática transcutânea sincronizada com o sinal respiratório, onde foram reavaliados depois de 10 sessões de 20 minutos, utilizando os mesmos parâmetros empregados no início do protocolo (Figura 7).

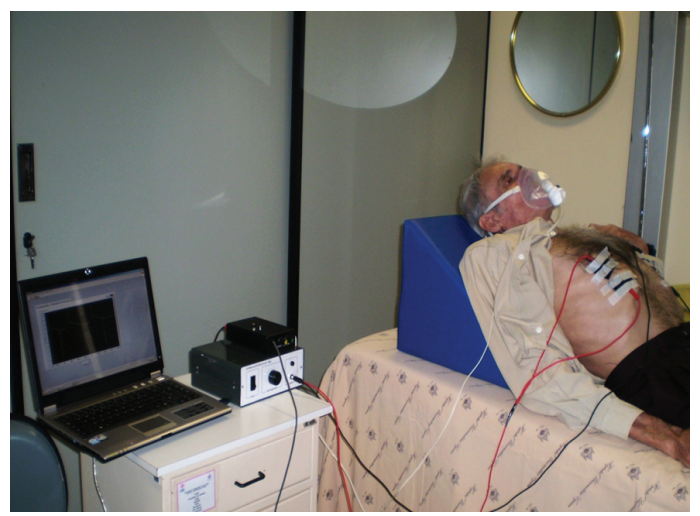

Figura 7. Foto ilustrando a aplicação do protocolo experimental de estimulação elétrica diafragmática sincronizada.

Figure 7. Photo illustrating the application of the experimental protocol of the transcutaneous diaphragmatic synchronized pacing.

\section{Resultados}

Os resultados obtidos na avaliação inicial e depois das 10 sessões estimulatórias, as diferenças entre os dois momentos, assim como a diferença média para a variável função pulmonar, avaliada por meio da espirometria não produziram significância estatística, os quais podem ser visualizados na Tabela 1 .
A variável força muscular, avaliada pela técnica manovacuometria, com medições realizadas antes e depois das sessões estimulatórias, apresentaram significância estatística, conforme indicam os valores inscritos na Tabela 2.

Os domínios referentes aos efeitos do programa de EEDS sobre a qualidade de vida, por meio do SGRQ (Questionário do Hospital St. George sobre problemas respiratórios), são apresentados na Tabela 3.

Para a qualidade de vida, constatou-se uma redução em todos os domínios avaliados pelo SGRQ. Essas variações negativas, obtidas após a aplicação do protocolo de estimulação elétrica sobre o SGRQ, mostraram-se significativas em todos os domínios, com a aplicação do teste não paramétrico de Willcoxon.

\section{Discussão}

Inúmeros são os protocolos encontrados na literatura para a detecção do sinal respiratório empregando sensores de fluxo (Sorli et al., 2004), sensores a fibras óticas (Ávila, 2001), sensores piezolétricos (Torres et al., 2004), aceletrômetros (Torres et al., 2006). No entanto, alguns desses métodos apresentam limitações práticas que restringem sua aplicabilidade, como relata Ávila (2001).

Neste trabalho, optou-se por termistores do tipo NTC, os quais possibilitaram a aquisição de sinal livre das interferências elétricas, originadas pelos pulsos aplicados nos pontos motores durante a fase de estimulação diafragmática. Com isso, tornou-se possível a obtenção de um sinal bem definido e com pouca interferência, eliminando assim, a necessidade de pós-processamento de sinal.

Os termistores NTC apresentaram sensibilidade adequada às pequenas variações de deslocamento de ar (consequentemente, de temperatura) e uma rápida resposta no tempo, viabilizando sua utilização para os

Tabela 1. Média, desvio padrão, diferença média e valor de $\mathrm{p}$ das variáveis obtidas através da espirometria $(\mathrm{N}=6)$.

Table 1. Mean, standard deviation, mean difference and p-value of the variables obtained by spirometry $(N=6)$.

\begin{tabular}{lllrc}
\hline \multicolumn{1}{c}{ Parâmetros } & Pré-EEDS & Pós-EEDS & Diferença & p \\
\hline CVF (l) & $2,77 \pm 1,18$ & $2,70 \pm 1,32$ & $-0,07 \pm 0,24$ & 0,345 \\
CVF\% do pred (\%) & $0,72 \pm 0,23$ & $0,70 \pm 0,28$ & $-0,02 \pm 0,11$ & 0,600 \\
VEF $_{1}$ (l) & $1,27 \pm 0,72$ & $1,40 \pm 1,00$ & $0,12 \pm 0,32$ & 0,600 \\
VEF $_{1}$ do pred (\%) & $0,43 \pm 0,22$ & $0,47 \pm 0,30$ & $0,03 \pm 0,11$ & 0,917 \\
VEF $_{1} /$ CVF (l) & $0,47 \pm 0,16$ & $0,49 \pm 0,17$ & $0,02 \pm 0,03$ & 0,109 \\
VEF $_{1} /$ CVF\% do pred (\%) & $0,60 \pm 0,20$ & $0,62 \pm 0,22$ & $0,02 \pm 0,04$ & - \\
\hline
\end{tabular}

CVF: capacidade vital forçada (1); CVF\% do pred (\%): capacidade vital forçada expressa em porcentagem dos valores previstos; $\mathrm{VEF}_{1}$ : volume expiratório forçado no primeiro segundo; $\mathrm{VEF}_{1} \%$ do pred (\%): volume expiratório forçado no primeiro segundo expresso em porcentagem dos valores previstos; $\mathrm{VEF}_{1} / \mathrm{CVF}(\%)$ : Índice de Tiffeneau: $\mathrm{VEF}, / \mathrm{CVF}$ do pred\%: Índide de Tiffeneau expresso em porcentagem dos valores previstos; EEDS: estimulação elétrica diafragmática sincronizada.

FVC: forced vital capacity (1), FVC\% of pred (\%): forced vital capacity expressed as a percentage of the predicted values, FEV : forced expiratory volume in the first second; $F E V_{1} \%$ of pred (\%): forced expiratory volume in one second expressed as percentage of the predicted values; $F E V_{1} / F V C(\%)$ :Tiffeneau Index: $F E V_{1} / F V C \%$ of pred: Tiffeneau Index expressed as percentage of predicted values; EEDS: transcutaneous diaphragmatic synchronized pacing 
Tabela 2. Média, desvio padrão, diferença média e valor de $\mathrm{p}$ das pressões inspiratórias $\left(\mathrm{PI}_{\text {max }}\right)$ e expiratórias $\left(\mathrm{PE}_{\max }\right)(\mathrm{N}=6)$. Table 2. Mean, standard deviation, mean difference and p-value of the inspiratory $\left(I P_{\max }\right)$ and expiratory $\left(E P_{\operatorname{mix}}\right)$ pressures $(N=6)$.

\begin{tabular}{lccccc}
\hline \multicolumn{1}{c}{ Parâmetros } & $\begin{array}{c}\text { Média dos } \\
\text { valores previstos }\end{array}$ & Pré-EEDTS & Pós-EEDTS & Diferença & Valor de p \\
\hline $\mathrm{PI}_{\text {máx }}\left(\mathrm{cmH}_{2} \mathrm{O}\right)$ & 97,77 & $66,67 \pm 12,11$ & $91,67 \pm 25,03$ & $25,00 \pm 13,78$ & 0,028 \\
$\mathrm{PE}_{\text {máx }}\left(\mathrm{cmH}_{2} \mathrm{O}\right)$ & 103,05 & $92,50 \pm 10,84$ & $116,67 \pm 8,16$ & $24,17 \pm 9,17$ & 0,028 \\
\hline
\end{tabular}

Significância estatística: $\mathrm{p}<0,05$. Valor de $\mathrm{p}$ calculado a partir do teste estatístico não-paramétrico de Wilcoxon.

Statistical significance: $p<0.05$. P-value calculated from the non-parametric Wilcoxon statistical test.

Tabela 3. Média, desvio padrão, diferença média e valor de p dos domínios sintomas, atividade funcional, impacto e escore total do questionário de qualidade de vida (SGRQ) $\operatorname{com} \mathrm{N}=6$.

Table 3. Mean, standard deviation, mean difference and p-value of symptoms, functional activity, impact domains and total score of quality of life questionnaire (SGRQ) with $N=6$.

\begin{tabular}{lcccc}
\hline \multicolumn{1}{c}{ Parâmetros } & Pré-EEDS & Pós-EEDS & Diferença & p \\
\hline Sintomas (\%) & $49,10 \pm 19,40$ & $28,60 \pm 25,20$ & $-20,50 \pm 24,40$ & 0,028 \\
Atividade (\%) & $83,40 \pm 12,50$ & $64,57 \pm 18,80$ & $-19,00 \pm 11,10$ & 0,028 \\
Impacto (\%) & $54,10 \pm 11,34$ & $38,00 \pm 27,07$ & $-16,15 \pm 19,52$ & 0,249 \\
Score total (\%) & $65,50 \pm 7,60$ & $44,47 \pm 22,31$ & $-21,08 \pm 19,13$ & 0,028 \\
\hline
\end{tabular}

Os domínios sintomas, atividade e impacto foram avaliados utilizando o St. George's Respiratory Questionnaire, os quais estão expressos em média, apresentando $\mathrm{p}<0,05$.

Symptoms, activity and impact domains were assessed using the St. George's Respiratory Questionnaire, which are expressed as mean, with $p<0.05$.

mais variados padrões respiratórios. O encapsulamento pequeno e robusto do sensor escolhido possibilitou sua fixação na máscara respiratória e permitiu fácil substituição e higienização. Comparando com o sensor à fibra óptica baseado em redes de Bragg do trabalho desenvolvido por Ávila (2001), constatou-se que o uso dos termistores eliminou a necessidade de cuidadosos ajustes e calibrações, além de apresentarem robustez mecânica. Apesar disso, o sinal adquirido com aquele sensor era mais imune a ruídos e interferências elétricas que o sistema empregado nesta pesquisa.

A visualização do sinal na tela de um computador tornou rápido o ajuste do sinal, que é realizado no início do procedimento com auxílio dos potenciômetros que controlam offset e ganho do amplificador, de acordo com a diferença de temperatura entre $\mathrm{o}$ ar expirado e ambiental. Além disso, possibilita um controle dos eventos por parte do profissional da área médica durante todo o tempo do procedimento.

A avaliação espirométrica fornece resultados quantitativos e reprodutíveis, permitindo avaliações longitudinais, o que permite aos profissionais da área da saúde e correlacioná-los à gravidade e à progressão de diversas doenças, de acordo com Pereira (2004). Também permite a avaliação de uma multiplicidade de parâmetros, porém, na aplicação clínica os parâmetros mais relevantes são a $\mathrm{CFV}, \mathrm{o} \mathrm{VEF}_{1}$ e a relação $\mathrm{VEF}_{1} /$ CVF, segundo a SBPT (Sociedade..., 2000).

No estudo realizado, não houveram alterações consideráveis dos parâmetros espirométricos avaliados pré e pós a aplicação do protocolo de EEDS, portanto, sem relevância estatística. Tal fato ocorre porque a atividade física, assim como os outros componentes da reabilitação pulmonar não proporcionam benefícios na função pulmonar, atuando apenas nos distúrbios secundários impostos pela DPOC, de acordo com Fishman (1994) e Panizzi (2004).

Rodrigues et al. (2003) afirmam que o comprometimento irreversível da arquitetura pulmonar, proveniente da DPOC, é a principal razão dos programas de reabilitação pulmonar não beneficiarem o quadro de obstrução ao fluxo aéreo. Contudo, a EEDS auxilia secundariamente na doença pulmonar, minimizando as disfunções musculares periféricas e respiratórias, anormalidades nutricionais, deficiências cardiovasculares, distúrbios esqueléticos, sensoriais e psicossociais. A EEDS não produziu melhoras significativas nos parâmetros espirométricos. Contudo, como apontado por Panizzi, (2004), esta técnica é uma importante ferramenta para determinação do grau de obstrução pulmonar.

Ao avaliar a força muscular inspiratória, por intermédio das pressões máximas inspiratória $\left(\mathrm{PI}_{\text {máx }}\right)$ e expiratória $\left(\mathrm{PE}_{\text {max }}\right)$, medidas por meio da manovacuometria, notou-se que todos os participantes apresentaram aumento significativo após a aplicação do protocolo de EEDS, em que a $\mathrm{PI}_{\text {máx }}$ sofreu um aumento médio de 66,67 para $91,67 \mathrm{cmH}_{2} \mathrm{O}$ e a $\mathrm{PE}_{\text {máx }}$ de 92,50 para $116,67 \mathrm{cmH}_{2} \mathrm{O}$.

De acordo com Paulin (2006), existem evidências de que o diafragma seja o músculo que apresenta maior comprometimento em pacientes hiperinsuflados. Este fato, além de promover alterações de volumes e da capacidade pulmonar, interfere também na atuação, 
mobilidade e conformidade do diafragma, que tende a se retificar diminuindo sua zona de aposição, fazendo com que haja limitação ventilatória. Além disso, estudos de Gosselink et al. (1996), sugerem que a diminuição da força muscular ventilatória esteja relacionada à hipercapnia, dispnéia, limitação da capacidade de física e, consequentemente, à qualidade de vida dos portadores de DPOC.

Sabe-se que o treinamento dos músculos ventilatórios diminui a sensação de dispnéia e aumenta a endurance dos músculos respiratórios, de acordo com Casals (2005), Dekhuijzen et al. (1991), Riera et al. (2001), Sturdy et al. (2003) e Wanke et al. (1994). Portanto, o aumento da $\mathrm{PI}_{\text {máx }}$ pós intervenção de EEDS apresenta resultados promissores na sensação de dispnéia, na capacidade funcional e na qualidade de vida.

$\mathrm{O}$ aumento significativo da $\mathrm{PE}_{\text {máx }}$ após as dez aplicações de EEDS pode ser justificado devido à adaptação muscular expiratória frente às alterações mecânicas dos músculos ventilatórios, provenientes da hiperinsuflação pulmonar, pois em pacientes obstrutivos crônicos, segundo Gáldiz Iturri (2000), a contração da musculatura abdominal desencadeia a redução do diâmetro das cúpulas diafragmáticas, otimizando a relação comprimento versus tensão do diafragma.

No decorrer das dez sessões, todos os sinais vitais verificados no início e ao término, tais como a pressão arterial, a frequência respiratória, a frequência cardíaca e a saturação de oxigênio apresentaram variações diárias de acordo com o estado físico e psicológico de cada paciente, apesar de consideradas estáveis.

Além disso, ao se comparar os sinais vitais antes e após a EEDS, percebeu-se uma diminuição da frequência cardíaca; confirmando, assim, que a técnica utilizada é um método seguro, não promovendo danos físicos nem possíveis alterações cardíacas (Riscilli et al., 1989). Acredita-se que tal evento tenha ocorrido devido a um possível relaxamento durante os 20 minutos de sessão.

Também verificou-se que todos os pacientes tiveram redução progressiva da FR (antes-depois de aplicado o protocolo experimental), indicando que a EEDS pode proporcionar momentaneamente uma redução da demanda ventilatória. A literatura revela que nos protocolos de tratamento em que há treinamento físico, ocorre um aumento da frequência respiratória, como consequência, a diminuição do tempo expiratório e uso da musculatura acessória, a fim de vencer o aumento da resistência das vias aéreas (West, 1996), tornando-se evidentes os benefícios proporcionados aos voluntários com DPOC deste estudo.
Após os atendimentos, constatou-se aumento de $\mathrm{SatO}_{2}$, levantando a hipótese de que a EEDS pode melhorar temporariamente o aporte de oxigênio na corrente sanguínea, proporcionando aos pacientes uma sensação de bem estar e diminuição do sintoma de dispnéia, o que é de se esperar em programas de reabilitação pulmonar convencionais (Sociedade..., 2000). Tal fato pode ser revisto nas avaliações pré e pós EEDS da dispnéia, através da escala de Borg Modificada, que apresentaram redução perceptível quando se considera os valores pré e pós-EEDS.

Os principais questionários utilizados em pneumopatas são o Questionário Respiratório Crônico (QRC) e o Questionário de Doenças Respiratórias do Hospital St. George (SGRQ). Embora o QRC seja altamente específico, os resultados numéricos fornecidos são difícies de processar quando estatísticamente. Já o SGRQ, segundo a SBPT (Sociedade..., 2000), permite comparações diretas.

Ao comparar os escores pré e pós-EEDS, verifica-se que os participantes reduziram em média $20,50 \%$ do domínio sintoma; $19,00 \%$ para o domínio atividade; $16,15 \%$ para o domínio impacto; e $21,08 \%$ para o escore total. Desta forma, a diferença entre e a avaliação antes-depois da aplicação do protocolo de EEDS, mostrou-se importante para os domínios sintomas e atividade, denotando significância estatística $(\mathrm{p}<0,028)$. Já no domínio impacto, constatou-se diminuição de $16,15 \%$, porém, sem significância estatística. Tal fato revela que o protocolo de EEDS atuou diretamente na sintomatologia e na atividade funcional dos voluntários portadores de DPOC, não interferindo de forma significativa no domínio impacto da doença sobre os pacientes.

Apesar de terem sido triados dezoito voluntários (número similar a de outros estudos), tendo em vista os critérios de inclusão e exclusão definidos, além disponibilidade e motivação em aderir ao estudo experimental proposto, assim como o atraso ocorrido entre o processo de triagem e o início da aplicação clínica (decorrentes de entraves técnicos e logísticos), o número efetivo de participantes reduziu-se a seis, comprometendo a viabilidade de formação de dois grupos de estudo: teste e controle; assim como a realização de avaliação cega. Evidentemente, isso torna os resultados limitados, mas mesmo assim sugerem uma perspectiva promissora de aplicação dessa técnica estimulatória em pessoas com DPOC, tendo em vista que outros estudos realizados também apresentaram limitações semelhantes. Gollee et al. (2007) aplicaram estimulação elétrica na musculatura abdominal de apenas quatro voluntários tetraplégicos, visando melhorar a função respiratória, e obtiveram aumentos de volume corrente (entre 9 e $71 \%$ da linha 
de base) e pico de fluxo da tosse (entre 31e 54\% da linha de base), o que levou os autores a concluírem pelo sucesso da técnica. Sorli et al. (1994) estudaram a viabilidade de estimular eletricamente os músculos abdominais durante a ventilação normal em cinco voluntários sadios. Verificaram que, desencadeando estimulação sincronizada ao fluxo de ar expiratório na boca, o volume corrente aumentou. Torry et al. (1996) estudaram os efeitos da estimulação elétrica nervosa transcutânea sobre a parede torácica em situação de falta de ar em onze pessoas normais durante o exercício e concluíram que nas circunstâncias avaliadas, a estimulação elétrica não produziu efeito sobre a sensação de falta de ar ou nas variáveis fisiológicas medidas em indivíduos normais durante o exercício. Independentemente dos resultados, nesses três estudos, não houve grupo controle nem avaliação cega.

Vivodtzev et al. (2006) avaliaram os benefícios produzidos pela estimulação elétrica em programas de reabilitação para pacientes com DPOC severa sem condicionamento físico e após exacerbação aguda. Aplicaram o protocolo em um grupo maior, contendo dezessete voluntários, num estudo controlado e randomizado e concluíram que os resultados do grupo teste empregando reabilitação tradicional associada à estimulação elétrica, comparados à reabilitação tradicional exclusivamente, foram muito superiores estatisticamente no que tange à função muscular e à redução da dispnéia. Esses resultados, portanto, corroboram com os obtidos na pesquisa descrita neste artigo e, por extensão, na estratégia empregada com número limitado de participantes. Um número maior de voluntários viria a ressaltá-los, evidentemente.

\section{Conclusões}

$\mathrm{O}$ instrumento desenvolvido utilizando sensores de temperatura NTC mostrou-se adequado para a detecção das fases respiratórias e para sincronismo de equipamentos de estimulação elétrica neuromuscular.

A interface gráfica desenvolvida facilitou o acompanhamento de cada paciente pelo staff clínico durante as sessões, proporcionando a percepção dos fenômenos ocorridos durante da estimulação elétrica, tais como o momento de liberação de cada estímulo e a frequência respiratória.

A aplicação da estimulação elétrica sincronizada promove aumento da força muscular diafragmática em pacientes com DPOC que apresentam disfunções diafragmáticas, por meio do recrutamento das fibras musculares, mesmo que os estímulos sejam aplicados por via transcutânea.

A estimulação sincronizada pelo sinal respiratório configura-se como uma opção promissora a ser aplicada em disfunções diafragmáticas, mostrando-se uma técnica simples mas eficaz. Apesar da amostra ser pequena, merece ser explorada em um estudo multicêntrico envolvendo um conjunto amostral mais significativo.

\section{Referências}

American Toracic Society-ATS. Statement: standards for the diagnosis and care of patients with chronic obstrutive pulmonary disease. American Journal of Respiratory and Critical Care Medicine. 1995; 152(1):77-120.

Ávila JLS. O emprego da estimulação elétrica transcutânea no tratamento da disfunção diafragmática [dissertação]. Curitiba: Universidade Tecnológica Federal do Paraná; 2001.

Azeredo CAC. Fisioterapia Respiratória Moderna. 3. ed. São Paulo: Manole; 1999.

Biaggi AL, Silva JL, Ferrazza F, Nohama P. Sistema de estimulação elétrica para prevenção e tratamento de disfunção diafragmática. In: CBEB 2000: Anais do $17^{\circ}$ Congresso Brasileiro de Engenharia Biomédica; 2000 set. 11-13; Florianópolis, Brasil. Florianópolis; 2000. p. 560-3.

Back LF, Hyatt RE. Maximal respiratory pressures: normal values and relationship to age and sex. American Review Respiratory Disease. 1969; 99(5):696-702. PMid:5772056.

Camelier A, Rosa FW, Salmi C, Nascimento AO, Cardoso F, Jardim JR. Avaliação da qualidade de vida pelo questionário do Hospital Saint George na doença respiratória em portadores de doença pulmonar obstrutiva crônica: validação de uma nova versão para o Brasil. Jornal Brasileiro de Pneumologia. 2006; 32(2):114-22. PMid:17273580. http:// dx.doi.org/10.1590/S1806-37132006000200006

Cancelierro KM, Costa D, Silva CA. Estimulação diafragmática elétrica transcutânea melhora as condições metabólicas dos músculos respiratórios de ratos. Revista Brasileira de Fisioterapia. 2006; 10(1):59-65.

Casals SM. What is the role of inspiratory muscle training in the treatment of chronic obstructive pulmonary disease? Archivos de Bronconeumología. 2005; 41(11):593-5. http:// dx.doi.org/10.1016/S1579-2129(06)60291-7

Celli BR, MacNee W. Standards for the diagnosis and treatment of patients with COPD: a summary of the ATS/ERS position paper. European Respiratory Journal. 2004; 23:932-46. PMid:15219010. http://dx.doi. org/10.1183/09031936.04.00014304

Costa D. Fisioterapia Respiratória Básica. São Paulo: Atheneu; 1999.

Dekhuijzen PN, Folgering HT, Herwaarden CL. Target-flow inspiratory muscle training during pulmonary rehabilitation in patients with COPD. Chest. 1991; 1(99):128-33. PMid:1984942. http://dx.doi.org/10.1378/chest.99.1.128

Díez JM, Alonso I, París M, Cano B, Moro RG, Ramos L. Factors Affecting Drug Prescription in Patients Whit Stable COPD: results from a Multicenter Spanish Study. Archivos de Bronconeumología. 2005; 41(2):63-70. http:// dx.doi.org/10.1016/S1579-2129(06)60399-6 
Dourado VZ, Antunes LCO, Carvalho LR, Godoy I. Influência de características gerais da qualidade de vida de pacientes com doença pulmonar obstrutiva crônica. Jornal Brasileiro de Pneumologia. 2004; 30(2):207-14. http://dx.doi.org/10.1590/ S1806-37132004000300005

Dourado VZ, Godoy I. Recondicionamento muscular na DPOC: principais intervenções e novas tendências. Revista Brasileira de Medicina Esportiva. 2004; 10:331-4.

Fishman AP. Pulmonary rehabilitation research. American Journal of Respiratory and Critical Care Medicine. 1994; 149:825-33. PMid:8118655.

Fiz JA, Montserrat JM, Picado C. How many maneuvers should be done to measure maximal inspiratory mouth pressure in patients with chronic air-flow obstruction? Thorax. 1989; 44:419-21. PMid:2763242. PMCid:461850. http://dx.doi.org/10.1136/thx.44.5.419

Forti EMP, Pachani GP, Montebelo MIL, Costa D. Eletroestimulação diafragmática transcutânea em indivíduos saudáveis. Fisioterapia Brasil. 2005; 6(4):261-4.

Gáldiz Iturri JB. Función de los músculos respiratorios em la EPOC. Archivos de Bronconeumología. 2000; 36:275-85. PMid:10916667.

Geddes LA, Voorhees WD, Lagler R, Riscili CE, Foster K, Bourland JD. Electrically Produced Artificial Ventilation. West Lafayette: Medical Instrumentation. 1988; 22(5):263-71. PMid:3054441.

Global Iniciative For Chronic Obstrutive Lung Disease - GOLD. Global strategy for the diagnosis, management, and prevention of chronic obstrutive pulmonary disease - NHLBI/WHO Workshop Report. American Journal of Respiratory and Critical Care Medicine. 2001; 163(5):1256-76. PMid:11316667.

Global IniciativeFor Chronic Obstrutive Lung Disease - GOLD. Global strategy for the diagnosis, management and prevention of chronic obstructive pulmonary disease. GOLD; 2006. p. 1-88.

Gollee H, Hunt KJ, Allan DB, Fraser MH, McLean AN. A control system for automatic electrical stimulation of abdominal muscles to assist respiratory function in tetraplegia. Medical Engineering \& Physics. 2007; 29:799-807. http:// dx.doi.org/10.1016/j.medengphy.2006.08.007

Gosselink R, Troosters T, Decramer M. Peripheral muscle weakness contributes to exercise limitation in COPD. American Jounal of Respiratory and Critical Care Medicine. 1996; 153(3):976-80. PMid:8630582.

Hausson PM, Celli BR. Bilateral diaphragm paralysis secondary to central von recklighausen's disease. Chest. 2000; 117(4):1196-9. PMid:10767261.

Jardim Junior, Jones P, Sousa TC. Validação do questionário do hospital Saint George na doença pulmonar obstrutiva crônica do Brasil. Journal de Pneumologia. 2000; 26(3):119-28.

Knudson RJ, Lebowitz MD, Holberg CJ,Burrows B. Changes in the normal expiratory flow - volume curve growth and aging. American Review of Respiratory Disease. 1983; 127(6):725-34. PMid:6859656.
Kunikoshita LN, Silva YP, Silva TLP, Costa D, Jamami M. Efeitos de três programas de fisioterapia respiratória (PFR) em portadores de DPOC. Revista Brasileira de Fisioterapia. 2006; 10(4):449-55.

Miravitles M. Avaliação econômica da doença pulmonar obstrutiva crônica e de suas agudizações: aplicação na América Latina. Jornal Brasileiro de Pneumologia. 2004; 30(3):274-85.

Neder JA, Andreoni S, Castelo-Filho A, Nery LE. Reference values for lung function tests. I. Static volumes. Brazilian Journal of Medical Biological Research. 1999; 32(6):703-17. http://dx.doi.org/10.1590/S0100-879X1999000600006

Nishimura K, Izumi T, Tsukino M, Oga T. Dyspnea is a better predictor of 5- year survival than airway obstruction in patients with COPD. Chest. 2002; 121(5):1434-40. PMid:12006425. http://dx.doi.org/10.1378/chest.121.5.1434

Panizzi EA. Avaliação de um programa de reabilitação pulmonar de curta duração para portadores de doença pulmonar obstrutiva crônica [tese]. Curitiba: Pontifícia Universidade Católica do Paraná; 2004. 167 p.

Paulin E. Influência da mobilidade diafragmática na capacidade de exercícios e dispnéia em pacientes portadores de doença pulmonar obstrutiva crônica [tese]. São Paulo: Universidade de São Paulo; 2006.

Pereira CAC. Bases e aplicações clínicas dos testes de função pulmonar. Revista Brasileira de Medicina. 2004; 2(4):317-30.

Riera HS, Rubio TM, Ruiz FO, Ramos PC, Otero DDC, Hernandez TE, Gomez JC. Inspiratory muscle training in patients with COPD: Effect on dyspnea, exercise performance, and quality of life. Chest. 2001; 120(3):748-56. PMid:11555505. http://dx.doi.org/10.1378/chest.120.3.748

Riscilli CE, Hinds M, Voorhees WD, Bourland JD, Geddes LA. The safety factor for electroventilation measured by production of cardiac ectopy in the anesthetized dog. Chest. 1989; 95(1): 214-217. PMid:2909338. http://dx.doi. org/10.1378/chest.95.1.214

Rodrigues SL. Avaliação do programa de reabilitação pulmonar. In: Rodrigues SL. Reabilitação Pulmonar: conceitos básicos. São Paulo: Editora Manole; 2003. p. 135-48.

Sarmiento-Ramírez A, Levi-Orozco M, Guell R, Barreiro E, Hernandez N, Mota S, Sangenis M, Broquetas JM, Casan P, Gea J. Inspiratory muscle training in patients with chronic obstructive pulmonary disease: structural adaptation and physiologic outcomes. American Journal of Respiratory and Critical Care Medicine. 2002; 166:1491-7. PMid:12406842. http://dx.doi.org/10.1164/rccm.200202-075OC

Sociedade Brasileira de Pneumologia e Tisiologia - SBPT. I Consenso brasileiro de doença pulmonar obstrutiva crônica (DPOC). Journal Brasileiro de Pneumologia. 2000; 6(1):1-51.

Sorli J, Kandare F, Jaeger R, Stanic U. Ventilatory assistance using electrical stimulation of abdominal muscles. IEEE Transactions on Rehabilitation Engineering. 1994; 4(1):1-6.

Sousa TC, Jardim F, Jones P. Validação do questionário do Hospital Saint George na doença respiratória (SGRQ) em pacientes portadores de doença pulmonar obstrutiva crônica no Brasil. Journal Brasileiro de 
Pneumologia. 2000; 26(3):119-28. http://dx.doi.org/10.1590/ S0102-35862000000300004

Sturdy G, Hillman D, Green D, Jenkins S, Cecins N, Eastwood P. Feasibility of high-intensity, interval-based respiratory muscle training in COPD. Chest. 2003; 123(1):142-50. PMid:12527615. http://dx.doi.org/10.1378/chest.123.1.142

Torres A, Fiz JA, Galdiz B, Gea J, Morera J, Jané R. Assessment of respiratory muscle effort studying diaphragm movement registered with surface sensors. Animal model (dogs). In: ADCOM: Proceedings of the 26th Annual International Conference of the IEEE Engineering in Medicine and Biology Society; 2004 Sep 1-5; San Francisco, USA. San Francisco; 2004; 2. p. 3917-20.

Torres A, Fiz JA, Galdiz B, Gea J, Morera J, Jané R. Inspiratory pressure evaluation by means of the entropy of respiratory mechanomyographic signals. In: EMBS '06: Proceedings of the 28th Annual International Conference of the IEEE; 2006 Aug 30-Sep 3; New York City, USA. Engineering in Medicine and Biology Society; 2006; 30(3). p. $5735-8$.

Torry M, Wilcock A, Cooper BG, Tattersfield AE. The effect of chest wall transcutaneous electrical nerve stimulation on dyspnea. Respiration Physiology. 1996; 104:23-8. http:// dx.doi.org/10.1016/0034-5687(95)00100-X

Valenga MH, Jorge RF, Dos Santos AP, Schneider Junior B, Nohama P. Sistema de estimulação elétrica gatilhado por sinal respiratório. In: CBEB: Anais do $21^{\circ}$ Congresso Brasileiro de Engenharia Biomédica; 2008 nov. 16-20; Salvador. Salvador; 2008. p. 495-499.

Vivodtzev I, Pépin JL, Vottero G, Mayer V, Porsin B, Lévy P, Wuyam B. Improvement in quadriceps strength and dyspnea in daily tasks after 1 month of electrical stimulation in severely deconditioned and malnourished COPD. Chest. 2006; 29(6):1540-8. PMid:16778272. http:// dx.doi.org/10.1378/chest.129.6.1540

Wanke T, Formanek D, Lahrmann H, Brath H, Wild M, Wagner C, Zwich H. Effects of combined inspiratory muscle and cycle ergometer training on exercise performance in patients with COPD. European Respiratory Journal. 1994; 7:2205-11. PMid:7713205. http://dx.doi.or g/10.1183/09031936.94.07122205

Webster JG. Medical instrumentation: application and design. 3th ed. Wiley\& Sons; 1998.

West JB. Fisiologia respiratória moderna. 5th ed. São Paulo: Manole; 1996.

Yamaguti WPS, Paulin E, Slage JM, Chammas MC, Cukier A, Carvalho CRF. Diafragmatic dysfunction and mortality in patients with CPOD. Jornal Brasileiro de Pneumologia. 2009; 35(12):1174-81. PMid:20126918. http:/ dx.doi.org/10.1590/S1806-37132009001200003

\footnotetext{
Autores

Percy Nohama*

Programa de Pós-Graduação em Engenharia Elétrica e Informática Industrial - CPGEI, Laboratório de Engenharia de Reabilitação, Universidade Tecnologia Federal do Paraná - UTFPR, Av. Sete de setembro 3165, CEP 80230-901, Curitiba, PR, Brasil. Programa de Pós-Graduação em Tecnologia em Saúde - PPGTS, Pontifícia Universidade Católica do Paraná - PUCPR, Rua Imaculada Conceição, 1155, Prado Velho, CEP 80215-901, Curitiba, PR, Brasil.
}

\section{Rafaela Fernanda Jorge}

Programa de Pós-Graduação em Tecnologia em Saúde - PPGTS, Pontifícia Universidade Católica do Paraná - PUCPR, Curitiba, PR, Brasil.

\section{Marcelo Henrique Valenga}

Programa de Pós-Graduação em Engenharia Elétrica e Informática Industrial - CPGEI, Universidade Tecnologia Federal do Paraná - UTFPR, Curitiba, PR, Brasil. 